
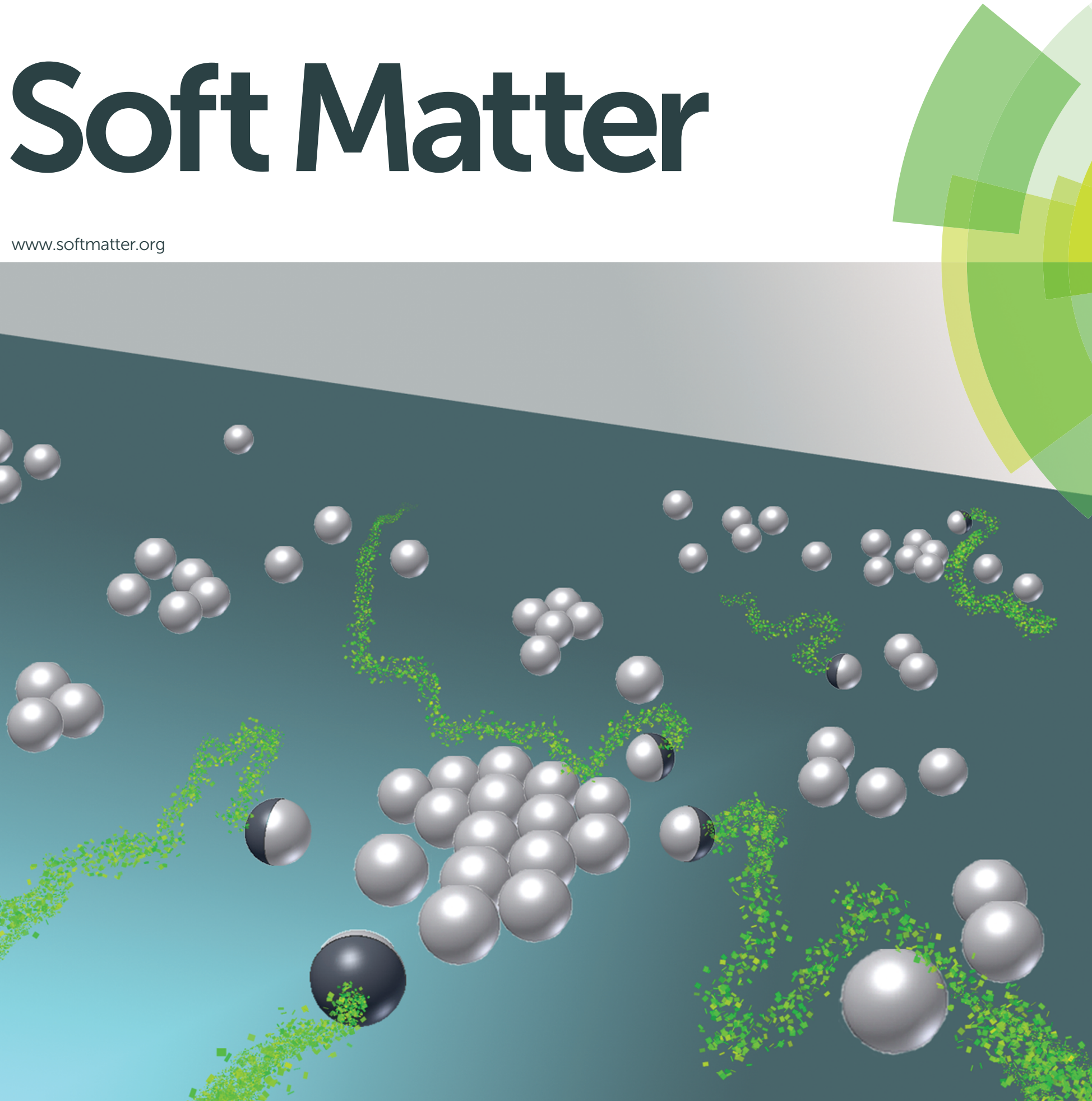


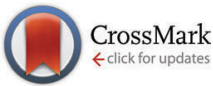

Cite this: Soft Matter, 2015, 11,6187

Received 8th April 2015, Accepted 25th June 2015

DOI: $10.1039 / \mathrm{c} 5 \mathrm{sm} 00827 \mathrm{a}$

www.rsc.org/softmatter

\section{Formation, compression and surface melting of colloidal clusters by active particles $\dagger$}

\author{
Felix Kümmel, ${ }^{a}$ Parmida Shabestari, ${ }^{a}$ Celia Lozano, ${ }^{b}$ Giovanni Volpe ${ }^{c d}$ and \\ Clemens Bechinger*ab
}

We demonstrate with experiments and numerical simulations that the structure and dynamics of a suspension of passive particles is strongly altered by adding a very small $(<1 \%)$ number of active particles. With increasing passive particle density, we observe first the formation of dynamic clusters comprised of passive particles being surrounded by active particles, then the merging and compression of these clusters, and eventually the local melting of crystalline regions by enclosed active particles.

\section{Introduction}

Self-propelled particles currently receive considerable interest because they provide deep insights into the self-organization of systems that are intrinsically in a non-equilibrium state such as living matter. Although in a homogeneous environment the long-time dynamics of such microswimmers is diffusive, ${ }^{1}$ the properties of active particles strongly differ from those of (passive) Brownian objects when considering complex and crowded environments where mutual interactions between active particles ${ }^{2-9}$ or encounters with rigid obstacles ${ }^{10-13}$ become important. The presence of active particles can also alter the dynamics of single passive particles, e.g., by imposing a super-diffusive or even uni-directional motion onto the passive colloids. ${ }^{14,15}$ Most experiments with such mixtures have been carried out with highly diluted suspensions of passive particles. In contrast, only little is known about the situation when active particles interact with a dense background of passive colloids. Numerical simulations have predicted that, under such conditions, active particles can significantly promote the crystallization of (passive) hard-sphere systems, even at concentrations where the formation of crystals is hindered by kinetic trapping of particles in a glassy state. ${ }^{16-18}$ However, the effect of active particles on the dynamics of a dense suspension

\footnotetext{
${ }^{a}$ 2. Physikalisches Institut, Universität Stuttgart, D-70569 Stuttgart, Germany. E-mail: c.bechinger@physik.uni-stuttgart.de

${ }^{b}$ Max-Planck-Institut für Intelligente Systeme, D-70569 Stuttgart, Germany

${ }^{c}$ Soft Matter Lab, Department of Physics, Bilkent University, Ankara 06800, Turkey

${ }^{d}$ UNAM-National Nanotechnology Research Center, Bilkent University, Ankara 06800, Turkey

$\dagger$ Electronic supplementary information (ESI) available: Details of particle tracking, dynamics of cluster formation and comparison with numerical simulations. Supplementary videos SV1 and SV2 of active particles moving inside a dense colloidal suspension. See DOI: 10.1039/c5sm00827a
}

of passive particles has not been investigated experimentally so far.

Here, we demonstrate with experiments and numerical simulations that the structure and dynamics of a suspension of passive particles is strongly altered by adding a very small $(<1 \%)$ number of active particles. Above a minimum passive particle concentration, we observe the formation of isolated, dynamical clusters of passive colloids, which are surrounded by active particles. At higher concentrations, such activity-induced clusters start to merge and undergo further compression. When exceeding the threshold for spontaneous crystallization, active particles are found to accumulate at the interfacial regions between crystalline domains where they lead to surface melting.

\section{Experimental section}

To obtain mixtures of equally-sized active and passive colloidal particles, we use silica spheres with $4.23 \mu \mathrm{m}$ diameter. A small amount of them was coated by sputtering from one side with a carbon layer of about $10 \mathrm{~nm}$ thickness and suspended together with uncoated particles in a mixture of water and 2,6-lutidine at critical composition (28.6 mass percent of lutidine). ${ }^{19,20}$ Such mixtures exhibit a lower critical point at $T_{\mathrm{C}}=307 \mathrm{~K}$. When the temperature of the solution is set below the mixture's lower critical point, all particles perform a diffusive motion as confirmed by the linear time-dependence of their mean-square displacement (data not shown). However, when the entire sample is illuminated $\left(\lambda=532 \mathrm{~nm}, I \leq 5 \mu \mathrm{W} \mu \mathrm{m}^{-2}\right)$, the light is partially absorbed by the carbon caps. This leads to the local heating and demixing of the mixture near the caps, which generates a chemical gradient around the particle and eventually results in a self-diffusiophoretic particle motion. ${ }^{11,21}$ Since the local heating at the cap and, thus, the local concentration 
gradient depends on the illumination intensity, it is possible to alter in situ the particle's Peclet number $\mathrm{Pe}=v / \sqrt{D_{\mathrm{R}} \cdot D_{\mathrm{T}}}$, where $v$ is the swim velocity of an isolated particle, and $D_{\mathrm{T}}$ and $D_{\mathrm{R}}$ are its translational and rotational diffusion coefficients (for details regarding the propulsion mechanism $\mathrm{see}^{11,22}$ ). Since the uncapped colloids are transparent to light and thus present no absorption, their motion is not affected by the laser light. The interparticle forces are dominated by steric interactions, ${ }^{7}$ because electrostatic repulsion is very short-ranged (the electrostatic screening length of the liquid mixture is about $12 \mathrm{~nm}^{23}$ ) and van der Waals forces are negligible. Particle motion is confined to two dimensions by using sample cells with about $6 \mu \mathrm{m}$ in height. The sample cell surfaces are rendered negatively charged by a plasma treatment to reduce particle sticking. The particle trajectories are recoded by digital video microscopy at 7.5 frames per second. Due to their carbon coating, active particles appear darker than passive ones which provides sufficient optical contrast to distinguish them from the passive ones (see Fig. S1, ESI $\dagger$ ). The area fraction of passive particles $\eta_{\mathrm{p}}$ was varied between $\eta_{\mathrm{p}}=0.10$ and $\eta_{\mathrm{p}}=0.90$, while the area fraction of active particles was kept constant at $\eta_{\mathrm{a}} \approx 0.01$.

\section{Experimental results and discussion}

In Fig. 1 we illustrate the typical temporal changes in a colloidal suspension when interacting with a small number of active particles with $\mathrm{Pe} \approx 20$. In every set of experiments, first the sample is equilibrated over at least $60 \mathrm{~min}$ without illumination so that all particles behave as passive particles; this yields a homogeneous particle distribution at $t=0 \mathrm{~s}$ (Fig. 1(a)). Afterwards the illumination is turned on so that the active particles start performing active motion and colliding with nearby passive particles (trajectories of active particles are marked as

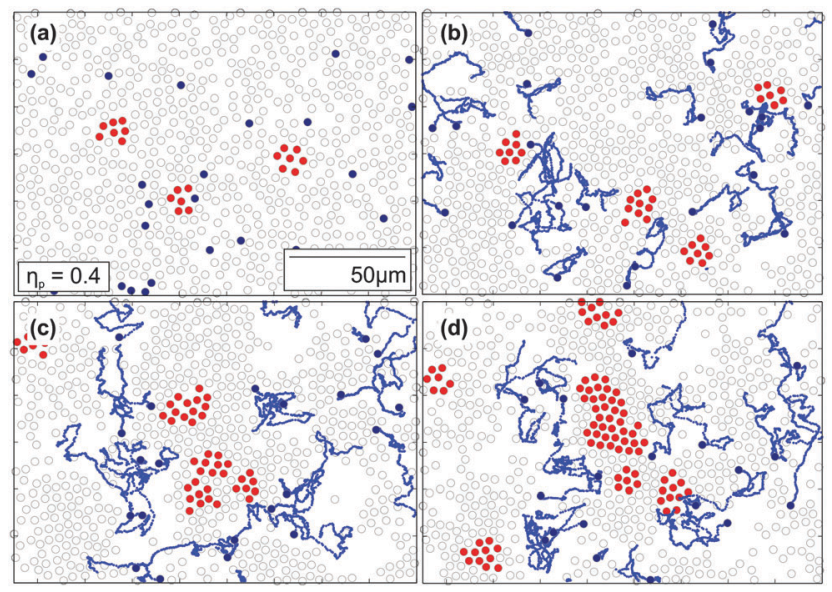

Fig. 1 Experimental snapshots of the temporal evolution of a mixture of passive $\left(\eta_{\mathrm{p}}=0.40\right)$ and active $\left(\eta_{\mathrm{a}} \approx 0.01\right)$ particles at (a) $0 \mathrm{~s}$, (b) $600 \mathrm{~s}$, (c) $900 \mathrm{~s}$ and $(\mathrm{d}) 1200 \mathrm{~s}$ for $\mathrm{Pe} \approx 20$. The passive particles belonging to clusters are represented as red circles, while those not belonging to clusters are represented as open circles. Active particles are shown as blue bullets and their trajectories over $300 \mathrm{~s}$ prior to each snapshot are represented as blue lines. blue lines in Fig. 1). Such collisions lead to the creation of depleted regions behind the active particles, to compression of the passive particles and, thus, to the generation of enhanced density variations. This is similar to what is observed when a single particle is forced by an external field through a dense colloidal suspension. ${ }^{24}$ Most importantly, however, active particles lead to the formation and compression of clusters of passive particles (marked in red in Fig. 1). A particle belonging to a cluster is defined as being six-fold coordinated and its next neighbor distances $d<7 \mu \mathrm{m}$. To reduce the effect of statistical density fluctuations, which would - even without active particles cause short-lived colloidal clusters, in our analysis we only considered clusters comprised of at least seven particles (typically, the life-time of such large clusters is more than several hundreds of seconds). As clearly seen from Fig. 1, active particles become increasingly localized at the boundaries of such clusters, which is in good agreement with recent numerical simulations. ${ }^{16,18}$

The effect of active particles on a suspension of colloids strongly depends on the packing fraction $\eta_{\mathrm{p}}$ (for a given value of Pe). In addition to enhanced crystallization, also the opposite, i.e. local melting induced by active particles, can be observed. Fig. 2 compares configurational snapshots of the system for increasing $\eta_{\mathrm{p}}$ in equilibrium at $t=0 \mathrm{~s}$ (left column) and steady state after illumination at $t=1200 \mathrm{~s}$ (right column). The color code of passive particles within clusters corresponds to their bond orientation, which is derived from the complex six-fold bond-orientational order parameter

$$
\psi_{6}\left(\mathbf{r}_{j}\right)=\frac{1}{6} \sum_{k=1}^{6} \exp \left(6 i \theta\left(\mathbf{r}_{j k}\right)\right),
$$

where $\theta\left(\mathbf{r}_{j k}\right)$ is the angle between a particle $j$ and its next neighbor $k$ relative to a reference axis. The phase of $\psi_{6}\left(\mathbf{r}_{j}\right)$ corresponds to the bond orientation of the neighbors around particle $j$, while its magnitude measures deviations from perfect hexagonal order. ${ }^{25}$

For $\eta_{\mathrm{p}} \leq 0.32$ (Fig. 2(a) and (b)), no significant changes in the system due to the presence of active particles are observed. This is confirmed by the pair correlation function, which remains almost unchanged (data not shown). At $0.32 \leq \eta_{\mathrm{p}}<$ 0.48 (Fig. 2(c) and (d)), we find that active particles induce clusters formed by passive particles as already shown in Fig. 1. To quantify this process, we calculated the time dependence of the number of clusters $N_{\mathrm{C}}$ (within our field of view) which is plotted by the dotted line in Fig. 3(a). After an initial strong increase, $N_{\mathrm{C}}$ levels off after about $300 \mathrm{~s}$ but still exhibits strong fluctuations afterwards which reflect the dynamic nature of the clusters. In addition, we measured the corresponding probability distributions of $N_{\mathrm{C}}$ for different time-intervals which are shown as inset in Fig. 3(b). Clearly, the distributions converge above $300 \mathrm{~s}$ which suggests that the system has reached a steady state during our experiments. This is further supported by calculating the associated complementary cumulative distribution functions $P(\mathrm{NC})$ where $\mathrm{NC} \geq N_{\mathrm{C}}$, i.e. the probability $P$ of finding at least $N_{\mathrm{C}}$ clusters in the field of view within the specified time interval. It has been demonstrated, that this quantity is rather robust against fluctuations ${ }^{26}$ (Fig. 3(b)). 

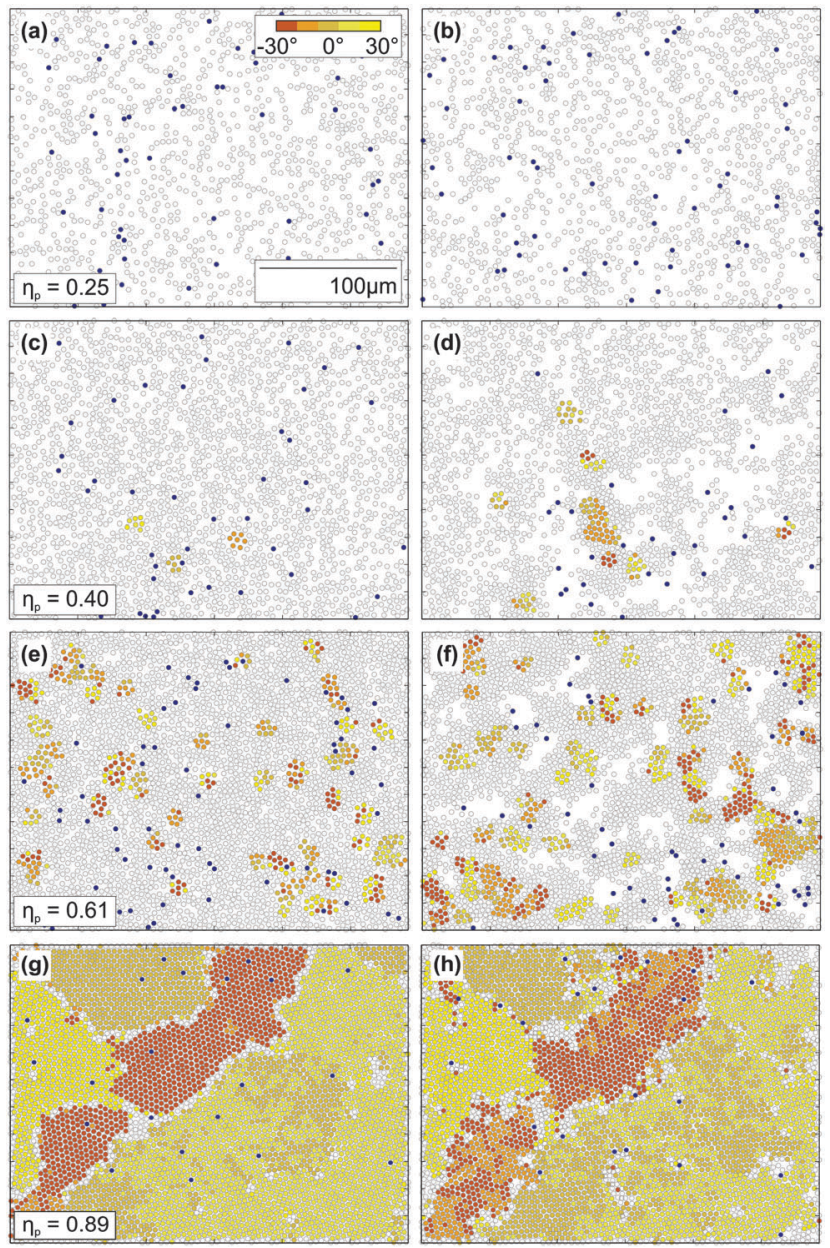

Fig. 2 Experimentally measured temporal change of the passive particle distribution for different values of $\eta_{\mathrm{p}}$ and constant $\mathrm{Pe} \approx 20$. The color code corresponds to the bond orientation of passive particles inside clusters (as defined in the text) relative to the horizontal axis. Passive particles not belonging to clusters are marked as open circles. Active particles are shown as blue bullets. (a) and (b) $\eta_{\mathrm{p}}=0.25$; (c) and (d) $\eta_{\mathrm{p}}=0.4$; (e) and (f) $\eta_{\mathrm{p}}=0.61$; (g) and (h) $\eta_{\mathrm{p}}=0.89$. (a), (c), (e) and (g) $t=0$; (b), (d), (f) and (h) $1200 \mathrm{~s}$.
At $0.48 \leq \eta_{\mathrm{p}}<0.62$ (Fig. 2(e) and (f)), activity-assisted crystallization becomes even more effective and leads to the formation of larger clusters. When comparing the temporal evolution of the CCDF, we find that, in this regime, the number of clusters decreases (Fig. 3(c)). This is due to the merging of smaller clusters into larger entities in agreement with Fig. 2(f).

Further increasing $\eta_{\mathrm{p}}$ eventually leads to crystallization of the colloidal suspension even in the absence of active motion. Such spontaneous crystallization is observed in our system above $\eta_{\mathrm{p}}>$ 0.62 , where we find a strong increase of the fraction of particles forming clusters. This is exemplarily seen in Fig. $2(\mathrm{~g})$ which corresponds to $\eta_{\mathrm{p}}=0.89$ and exhibits large crystalline domains. When active particles self-propel through crystalline domains, they locally melt the surrounding lattice and create defects along their trajectory. This is shown in Fig. 4(a) and (b) for an active particle initially located at a grain boundary and in Fig. 4(c) and 4(d) for a particle that is inside a previously defect-free crystalline region. When comparing the spatial extension of the trajectories (both corresponding to $1800 \mathrm{~s}$ ), it becomes obvious that active particles at grain boundaries are much faster than those moving inside a crystalline domain (compare also Videos SV1 and SV2, ESI $\dagger$ ). We attribute this to the large number of defects at grain boundaries which provide a lower resistance to the active particle motion. As a consequence, active particles show a tendency towards accumulation at grain boundaries because of the high stiffness of the adjacent crystalline regions. This is supported when comparing the positions of active particles prior (Fig. 2(g)) and after illumination (Fig. 2(h)). Accordingly, grain boundaries are particularly prone to activity-induced local melting, which leads to an increased width of disordered regions separating crystalline domains as seen in Fig. 4(b).

In total, we have prepared almost 50 samples where $\eta_{\mathrm{p}}$ was varied between 0.10 and 0.90 and the results are summarized in Table 1. Although small variations of the Peclet number do not change the qualitative behavior, a strong dependence of the system dynamics on Pe is found. This effect becomes particularly
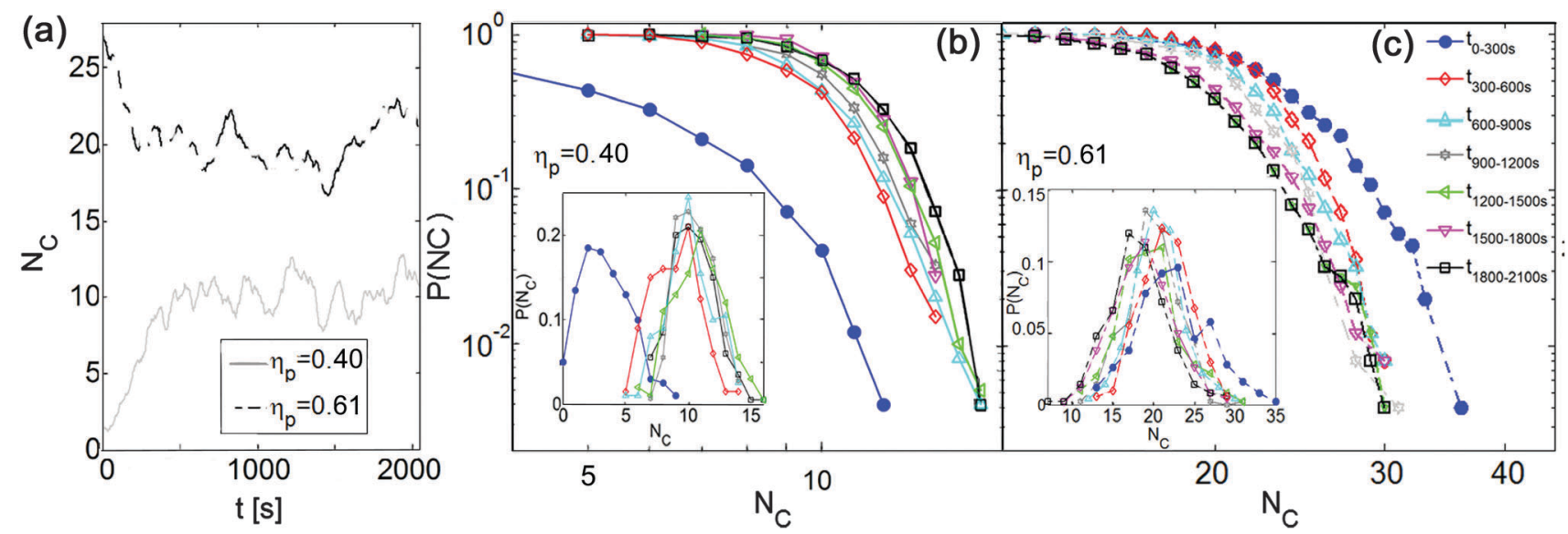

Fig. 3 (a) Experimentally measured number of clusters $N_{C}$ for $\eta_{p}=0.40$ and $\eta_{p}=0.61$. The data shown represents the moving average of $N_{C}$ over a defined number of time periods with $10 \mathrm{~s}$ length, each. (b) and (c) Experimentally measured Temporal evolution of the corresponding complementary cumulative distribution functions (CCDFs) of $N_{C}$ averaged over $300 \mathrm{~s}$ each for (b) $\eta_{\mathrm{p}}=0.40$ and (c) $\eta_{\mathrm{p}}=0.61$. The insets show the time-dependence of the probability distributions of $N_{C}$. The Peclet number in all plots is $\mathrm{Pe} \approx 20$. 

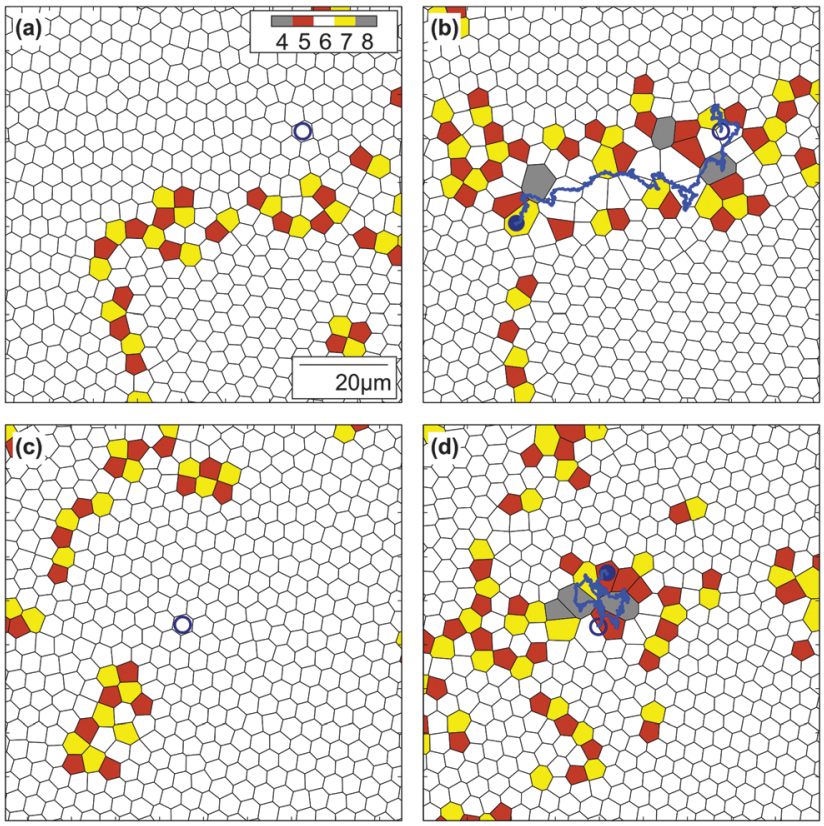

Fig. 4 Experimental trajectories of active particles (blue lines) along a grain boundary (a) and (b) and inside a crystalline domain (c) and (d) in a dense colloidal suspension with $\eta_{\mathrm{p}}=0.80$ and for $\mathrm{Pe} \approx 20$. Particle coordinates are represented by their Voronoi cells whose color code denotes the particle coordination number. Snapshots are taken at $t=0 \mathrm{~s}$ (a) and (c) and $t=1800 \mathrm{~s}$ (b) and (d) with the initial and final position of active particles marked with an open and filled circle, respectively.

pronounced at intermediate $\eta_{\mathrm{p}}$, where we observed a strong increase of the compression of clusters with increasing Pe (see Fig. S2, ESI $\dagger$ ). This indicates that not only the structure but also the system's dynamics is strongly modified by the presence of only few active particles.

\section{Numerical simulations}

In order to corroborate our experimental observations and to demonstrate the robustness and generic character of our findings, we have also performed computer simulations of a minimal model system. In our simulations we consider a twodimensional system of about 3000 Brownian particles with hard-sphere interactions in a square box with hard walls. The motion of the passive particles is modelled by two-dimensional Langevin equations, i.e.,

$$
\left\{\begin{array}{l}
\dot{x}_{i}(t)=\sqrt{2 D_{\mathrm{T}}} w_{x, i}(t) \\
\dot{y}_{i}(t)=\sqrt{2 D_{\mathrm{T}}} w_{y, i}(t)
\end{array}\right.
$$

where $x_{i}(t)$ and $y_{i}(t)$ are the coordinates of the $i$-th (passive) particle position, and $w_{x, i}(t)$ and $w_{y, i}(t)$ are two independent white noises. In addition to translational Brownian motion, a small fraction of particles is propelled with constant velocity along their cap orientation, which undergoes rotational diffusion, ${ }^{27}$ so that their corresponding equations of motion are

$$
\left\{\begin{array}{l}
\dot{x}_{j}(t)=v \cos \left(\varphi_{j}(t)\right)+\sqrt{2 D_{\mathrm{T}}} w_{x, j}(t) \\
\dot{y}_{j}(t)=v \sin \left(\varphi_{j}(t)\right)+\sqrt{2 D_{\mathrm{T}}} w_{y, j}(t) \\
\dot{\varphi}_{j}(t)=\sqrt{2 D_{\mathrm{R}}} w_{\varphi, j}(t)
\end{array}\right.
$$

where $x_{j}(t)$ and $y_{j}(t)$ are the coordinates of the $j$-th (active) particle position, $\varphi_{j}(t)$ is its orientation, $v$ is its speed, and $w_{x, j}(t), w_{y, j}(t)$ and $w_{\varphi, j}(t)$ are independent white noises. To account for the presence of surrounding particles and the walls of the sample cell, we introduce a short range $(<50 \mathrm{~nm})$ repulsion between the particles and the walls. Furthermore, we also account for the increased apparent viscosity in the presence of nearby particles due to hydrodynamic interactions. ${ }^{28}$ The densities, size, diffusion coefficients and Peclet numbers of the particles were chosen to match our experimental conditions.

In Fig. S3 and S4, ESI $\dagger$, we present the results of our simulations where we explore the effect of active particles on the dynamics of a dense suspension of passive particles finding good agreement with the experimentally observed regimes. In particular, the simulations reproduce the formation of clusters induced by the presence of active particles at lower packing fractions as well as the local melting and the accumulation of active particles at grain boundaries at higher packing fractions (Fig. S4, ESI $\dagger$ ). Furthermore, the simulation can also reproduce the evolution of the number of clusters over time, showing a decrease for $\eta_{\mathrm{p}}=0.61$ and an increase for $\eta_{\mathrm{p}}=0.50$ (Fig. S5(a), ESI $\dagger$ ) and the corresponding evolution of the CCDFs (Fig. S4(b) and (c), ESI $\dagger$ ). For very large simulation times and high particle densities, we observe the formation of large, defect-free colloidal domains. This is due to the already mentioned accumulation of active particles at grain boundaries and the permanent melting and recrystallization of such regions. This eventually leads to the macroscopic shift of grain boundaries and the growth of crystalline regions at expense of adjacent ones (Fig. S6, ESI $\dagger$ ). The overall good agreement between experiment and simulations also supports earlier findings that the diffusiophoretic propulsion mechanism employed in our experiments remains largely unaffected by the presence of other nearby particles. ${ }^{7}$

Table 1 Experimentally observed structural changes induced by a highly diluted suspension of active particles on a two-dimensional colloidal system with area fraction $\eta_{\mathrm{p}}$ and $\mathrm{Pe} \approx 20$

\begin{tabular}{ll}
\hline Packing fraction & State \\
\hline$\eta_{\mathrm{p}} \leq 0.32 \pm 0.02$ & No structural changes \\
$0.32 \pm 0.02<\eta_{\mathrm{p}} \leq 0.48 \pm 0.08$ & Induced cluster formation \\
$0.48 \pm 0.08<\eta_{\mathrm{p}} \leq 0.62 \pm 0.05$ & Compression \& merging of clusters \\
$0.62 \pm 0.05<\eta_{\mathrm{p}} \leq 0.89 \pm 0.01$ & Surface melting of crystalline domains
\end{tabular}




\section{Summary}

In summary, our experimental and numerical results show that doping of colloidal suspensions with a very small amount of active particles strongly influences the distribution and the dynamics of dense colloidal suspensions. Our study investigates how microswimmers behave in crowded environments resembling realistic situations under which active particles may be employed e.g. as drug delivery systems. In addition, our simulations suggest that the addition of active particles can lead to large defect-free crystalline domains which may suggest a novel route for the fabrication of high-quality colloidal crystals, which currently find use as sensors or photonic devices.

\section{Acknowledgements}

We acknowledge stimulating discussions with Ivo Buttinoni and technical support from Hans-Jürgen Kümmerer and Christa Mayer. This work is financially supported by the Deutsche Forschungsgemeinschaft within the Schwerpunktsprogramm Microswimmers SPP 1726.

\section{References}

1 J. R. Howse, R. A. Jones, A. J. Ryan, T. Gough, R. Vafabakhsh and R. Golestanian, Phys. Rev. Lett., 2007, 99, 048102.

2 J. Tailleur and M. Cates, Phys. Rev. Lett., 2008, 100, 218103.

3 A. Sen, M. Ibele, Y. Hong and D. Velegol, Faraday Discuss., 2009, 143, 15-27.

4 Y. Fily and M. C. Marchetti, Phys. Rev. Lett., 2012, 108, 235702.

5 I. Theurkauff, C. Cottin-Bizonne, J. Palacci, C. Ybert and L. Bocquet, Phys. Rev. Lett., 2012, 108, 268303.

6 J. Palacci, S. Sacanna, A. P. Steinberg, D. J. Pine and P. M. Chaikin, Science, 2013, 339, 936-940.

7 I. Buttinoni, J. Bialké, F. Kümmel, H. Löwen, C. Bechinger and T. Speck, Phys. Rev. Lett., 2013, 110, 238301.

8 A. Wysocki, R. G. Winkler and G. Gompper, Europhys. Lett., 2014, 105, 48004.
9 D. Levis and L. Berthier, Phys. Rev. E: Stat., Nonlinear, Soft Matter Phys., 2014, 89, 062301.

10 P. Galajda, J. Keymer, P. Chaikin and R. Austin, J. Bacteriol., 2007, 189, 8704-8707.

11 G. Volpe, I. Buttinoni, D. Vogt, H.-J. Kümmerer and C. Bechinger, Soft Matter, 2011, 7, 8810-8815.

12 N. Koumakis, A. Lepore, C. Maggi and R. Di Leonardo, Nat. Commun., 2013, 4, 2588.

13 A. Kaiser, H. Wensink and H. Löwen, Phys. Rev. Lett., 2012, 108, 268307.

14 X.-L. Wu and A. Libchaber, Phys. Rev. Lett., 2000, 84, 3017.

15 R. Di Leonardo, L. Angelani, D. Dell'Arciprete, G. Ruocco, V. Iebba, S. Schippa, M. Conte, F. Mecarini, F. De Angelis and E. Di Fabrizio, Proc. Natl. Acad. Sci. U. S. A., 2010, 107, 9541-9545.

16 R. Ni, M. A. Cohen Stuart and M. Dijkstra, Nat. Commun., 2013, 4, 2704.

17 R. Ni, M. A. Cohen Stuart, M. Dijkstra and P. G. Bolhuis, Soft Matter, 2014, 10, 6609-6613.

18 J. Stenhammar, R. Wittkowski, D. Marenduzzo and M. E. Cates, Phys. Rev. Lett., 2015, 114, 018301.

19 C. A. Grattoni, R. A. Dawe, C. Y. Seah and J. D. Gray, J. Chem. Eng. Data, 1993, 38, 516-519.

20 D. Beysens and D. Esteve, Phys. Rev. Lett., 1985, 54, 2123.

21 J. F. Brady, J. Fluid Mech., 2011, 667, 216-259.

22 B. ten Hagen, F. Kümmel, R. Wittkowski, D. Takagi, H. Löwen and C. Bechinger, Nat. Commun., 2014, 5, 4829.

23 C. Hertlein, L. Helden, A. Gambassi, S. Dietrich and C. Bechinger, Nature, 2008, 451, 172-175.

24 R. P. Dullens and C. Bechinger, Phys. Rev. Lett., 2011, 107, 138301.

25 P. Dillmann, G. Maret and P. Keim, Eur. Phys. J.: Spec. Top., 2013, 222, 2941-2959.

26 A. Clauset, C. Shalizi and M. Newman, SIAM Rev., 2009, 51, 661-703.

27 G. Volpe, S. Gigan and G. Volpe, Am. J. Phys., 2014, 82, 659-664.

28 H. Diamant, B. Cui, B. Lin and S. Rice, J. Phys.: Condens. Matter, 2005, 17, S2787. 